\title{
Lacunas de conhecimento e cooperação: análise de uma rede social em um instituto de tecnologia da região do $\mathrm{ABC}$ Paulista
}

\author{
Gaps of knowledge and cooperation: analysis of a social network in an institute \\ of technology in the $A B C$ Paulista region \\ Lacunas de conocimento y cooperación: análisis de una red social en un instituto
de tecnología en la región del ABC Paulista
}

Milton Carlos Farina
Rodrigo Santos de Melo
Flávio Gálio Araújo Dutra

Recebido em: Recebido em: 14/05/2020; revisado e aprovado em: 28/12/2020; aceito em: 30/12/2020 DOI: http://dx.doi.org/10.20435/inter.v22i3.3065

\begin{abstract}
Resumo: O objetivo da pesquisa foi analisar as relações entre empresas em rede organizacional, a partir do modelo de lacuna de conhecimento e cooperação. A pesquisa foi realizada em um instituto de tecnologia na região metropolitana de São Paulo. Os métodos utilizados foram a análise de redes sociais e a análise univariada da variância. O presente estudo mostrou que, individualmente, estar como diretor do instituto se faz relevante, embora, coletivamente, a redução da centralidade de poucos atores é compreendida como de grande importância.
\end{abstract}

Palavras-chave: análise de redes sociais; centralidade; lacunas do conhecimento; cooperação; instituto de tecnologia.

\begin{abstract}
This research aimed to analyze the relationships between companies in an organizational network based on the knowledge and cooperation gap model. The research was carried out at a technology institute in the metropolitan region of São Paulo. The methods used were social network analysis and univariate analysis of variance. The present study showed that, individually, being as director of the institute is relevant, although, collectively, the centrality reduction of few actors is viewed as of great importance.

Keywords: social network analysis; centrality; knowledge gaps; cooperation; institute of technology.

Resumen: El objetivo de la investigación fue analizar las relaciones entre empresas en una red organizacional, con base en el modelo de brecha de conocimiento y cooperación. La investigación se llevó a cabo en un instituto de tecnología en la región metropolitana de São Paulo. Los métodos utilizados fueron el Análisis de Redes Sociales y el Análisis de Varianza Univariante. El presente estudio mostró que estar como director individual del instituto es relevante, aunque, colectivamente, la reducción de la centralidad de pocos actores percibida como es de gran importancia.
\end{abstract}

Palabras clave: análisis de redes sociales; centralidad; brechas de conocimiento; cooperación; Instituto de Tecnología.

\section{INTRODUÇÃO}

As redes de inovação prosperam como organizações em três situações relacionadas ao conhecimento: i) está amplamente dispersa; ii) é complexo e iii) está em constante expansão (POWELL; KOPUT; SMITH-DOERR, 1996).

Uma vez que as redes organizacionais têm como principal objetivo a geração de conhecimento e inovação entre os atores, elas necessitam de cooperação entre seus participantes e são moldadas por interesses individuais e coletivos, que motivam principalmente pequenas e médias empresas (PMEs) a fazer parte de uma rede desta natureza (CÂMARA et al., 2018).

\footnotetext{
${ }^{1}$ Universidade Municipal de São Caetano do Sul (USCS), São Caetano do Sul, São Paulo, Brasil.

${ }^{2}$ Universidade Federal do Piauí (UFPI), Teresina, Piauí, Brasil.
} 
As redes de cooperação são projetadas para compartilhar conhecimento, informação e incentivar a criação de inovação (BRESCHI; MARLEBA, 2005). Estudos em ciências sociais aplicadas indicam que visões colaborativas e relacionais são importantes para as redes de cooperação, especificamente aquelas com objetivos de inovar e transferir conhecimento (CÂMARA et al., 2018).

Em contrapartida, considera-se a existência de redes, em que é possível identificar a presença das chamadas lacunas de conhecimento, evidenciando a sua relevância para a compreensão da dinâmica da rede organizacional (QIU; WANG; NIAN, 2014) em estudos na área de ciências sociais aplicadas.

Uma maneira de mapear a eficiência de uma rede em transferir conhecimento é por meio da consideração conjunta da sua densidade e da centralidade de seus atores. A alta densidade em uma rede cooperativa de inovação indica que as empresas cooperam estreitamente entre si para inovar, enquanto uma rede com baixa densidade mostra um fraco grau de cooperação entre seus membros (HANNEMAN; MARK, 2005), apontando para um baixo potencial inovador.

As redes são essencialmente ambientes apropriados para cooperação (CÂMARA et al., 2018; CAPALDO, 2007), na maioria das vezes construídas para inovar; mas, para que esta cooperação se consolide na rede, mecanismos de cooperação entre os atores devem ser incentivados (GUEDES et al., 2017; NOOTEBOOM; GILSING, 2005).

Dentro de uma rede, as empresas posicionadas centralmente são caracterizadas pelo aumento da transferência de conhecimento gerado internamente e pela maior capacidade de alavancar suas habilidades (DHANARAJ; PARKHE, 2006). Estas vantagens são obtidas por meio do maior número de vínculos, maiores oportunidades e maior poder para influenciar os demais atores (HANNEMAN; MARK, 2005). O fluxo de conhecimento, portanto, depende dos atores centrais, embora outros atores também tenham um papel fundamental na transferência de conhecimento (DHANARAJ; PARKHE, 2006).

A transferência de conhecimento pode ser otimizada pela gestão da rede, que fortalece a identidade comum dos membros (DHANARAJ; PARKHE, 2006), percebida como fundamental para incentivá-los a compartilhar conhecimento abertamente (DYER; NOBEOKA, 2000).

A estrutura de uma rede é decisiva para o alcance dos objetivos, como esclarecem Dhanaraj e Parkhe (2006), ao afirmar que, em algumas redes, uma estrutura dedicada à cooperação entre os membros pode priorizar os interesses do grupo e, por outro lado, poderá também reduzir a autonomia de empresas individuais. A partir dessa ambiguidade percebida nas relações dentro de uma rede organizacional, o presente estudo tem o objetivo de: analisar as relações entre empresas em rede organizacional, a partir do modelo de lacuna de conhecimento e cooperação, detalhado no quadro teórico.

Seguindo esta seção introdutória, o restante do artigo é dividido da seguinte forma. A segunda seção apresenta o quadro teórico que apoiou a construção desta pesquisa. Na terceira seção, apresenta-se a metodologia usada neste estudo e, na quarta seção, são realizadas as análises da rede, compostas pela Análise de Rede Social (ARS), Análise Univariada da Variância (ANOVA). A última seção apresenta as conclusões, limitações do estudo e sugestões para pesquisas futuras.

\section{QUADRO TEÓRICO}

A base teórica do presente estudo é dividida em três partes principais. Inicialmente, faz-se uma contextualização sobre a ARS e as principais medidas adotadas em seu estudo. No 
tópico seguinte, tem-se a abordagem sobre as possibilidades de como estas redes podem estar estruturadas, uma vez que a pesquisa foi desenvolvida em um ambiente de interconectividade entre diversas Empresas de Base Tecnológica. Por fim, a Rede Organizacional e suas lacunas de conhecimento, a partir do modelo desenvolvido por Qiu, Wang e Nian (2014).

\subsection{Análise de Rede Social (ARS)}

Como a maioria das empresas não tem todos os recursos necessários para alcançar seus objetivos, as relações cooperativas entre as organizações criam maior valor do que a relação competitiva entre elas, especialmente quando estão geograficamente mais próximas, pela possibilidade de geração de spillovers e externalidades positivas, e existe uma organização central, pela possibilidade da promoção de uma interação social (HITT; IRELAND; HOSKISSON, 2011).

Por sua vez, Zancan, Santos e Campos (2011) propõem que as redes sejam estruturadas com base na definição e alocação de papéis entre os atores, enquanto Nohria e Eccles (1992) argumentam que a rede é formada a partir da percepção de aspectos como interação e complementaridade entre os atores.

A rede social, portanto, é uma entidade complexa, devido ao aspecto dinâmico e ao aparente paradoxo para, por um lado, proporcionar integração entre seus atores e, por outro, buscar desempenho individual (BALESTRIN; VERSCHOORE; REYES, 2010).

Zancan, Santos e Campos (2011) dispensam especial atenção à importância de medir fenômenos relacionais e sugerir o uso de medidas de ARS para entender abordagens organizacionais entre duas ou mais empresas conectadas. Para os autores, as redes sociais estão constantemente se reconfigurando para adaptar melhor os recursos individuais e coletivos para alcançar o desempenho comum.

O objetivo do ARS nas áreas de gestão e redes organizacionais é entender a parceria entre os diversos membros em uma rede (LEONARDO et al., 2019). Essas relações podem ocorrer, por exemplo, por objetivos comuns ou proximidade espacial (COSTA et al., 2018) ou por uma melhor compreensão da questão da transferência e estabilidade do conhecimento (DHANARAJ; PARKHE, 2006).

Mais especificamente, estudos de redes sociais buscam entender as trocas de conhecimento entre membros da rede e a comercialização de produtos ou serviços, por meio dos nós e links (COSTA et al., 2018), e se esses links podem ser colaborativos e gerar algum produto inovador (AHUJA, 2000).

No presente artigo, a ARS é utilizada para identificar medidas estatísticas e representações gráficas do grau de densidade, que podem ser obtidas dividindo-se no número de possíveis vínculos na rede e no total de vínculos efetivamente ocorridos (HANNEMAN; MARK, 2005), de forma a indicar se há alta ou baixa interação entre empresas de rede, colaborando, assim, para o alcance do objetivo proposto.

Além dessa medida, o grau de centralidade é importante também para a ARS, por evidenciar o grau de visibilidade e atratividade dos atores (empresas) dentro da rede, por meio da quantidade de arestas, que podem ser ligações enviadas ou recebidas (HANNEMAN; MARK, 2005).

A centralidade é o número de atores com os quais cada ator da rede social está conectado (COSTA et al., 2018). A centralidade da rede divide-se em out-degree, que ocorre quando os atores da rede buscam conectar-se com os demais atores da rede, e o in-degree, que significa que os atores são procurados para que essa conexão se realize (COSTA et al., 2018). 
Outra medida importante na ARS é a chamada núcleo/periferia, que identifica o conjunto de atores com alta densidade de vínculos, sendo formada por aqueles que compartilham eventos comuns com maior frequência, chamados de núcleo, e o bloco formado por atores de baixo grau, caracterizado pela frequência de eventos desarticulados, chamados de periferia (LEONARDO et al., 2019; HANNEMANK; MARK, 2005).

Uma empresa central pode ser definida como a mais proeminente em uma rede (WASSERMAN; GALASKIEWICZ, 1994) e a que tem maior poder (BRASS; BURKHARDT, 1993), obtido por meio de características individuais e da disposição central na estrutura da rede. Portanto empresas que se posicionam de forma proeminente dentro da rede possuem maior poder para realizar a função de líder e agrupar recursos dispersos e capacidades dos atores da rede.

Hoppe e Reinelt (2010) acrescentam que, em uma rede organizacional, o líder pode se beneficiar ainda mais de sua própria posição, se a gestão compartilha informações e experiências e coopera principalmente com outras pessoas. Os autores constataram que, mesmo em uma análise de liderança em uma rede, o propósito em que as empresas foram inseridas e a razão pela qual a rede foi criada devem ser entendidos (HOPPE; REINELT, 2010).

Dhanaraj e Parkhe (2006) consideram a transferência do conhecimento como uma característica importante das empresas centrais, uma vez que é uma habilidade que avalia o conhecimento relevante que reside em diferentes pontos da rede e transfere-o para outras partes da mesma rede em que é percebido como necessário.

Considerando o propósito deste estudo, que é analisar as relações entre empresas em rede organizacional, a partir do modelo de lacuna de conhecimento e cooperação, a referência é feita ao pensamento de Zancan, Santos e Campos (2011) e Hoppe e Reinelt (2010), que consideram o SNA como a ferramenta mais apropriada para entender os fatores que impactam na formação de redes organizacionais.

\subsection{Estruturas de rede organizacional}

Grandori e Soda (1995) contribuíram para o estudo das redes para identificar três tipos básicos de redes organizacionais: (i) social, com a informalidade das relações intercompanhias como sua característica fundamental; (ii) burocracia, caracterizada pela existência de um acordo formal, pretendia regular mais do que as especificações de fornecimento (produto/serviço); e (iii) proprietários, o que se caracteriza pela formalização de acordos relativos aos direitos patrimoniais entre os acionistas das empresas.

Os autores supracitados identificaram que, além das relações formalizadas pela natureza do trabalho e pelos objetivos organizacionais, há de se considerar a presença da informalidade na forma como os membros de uma organização se relacionam entre si. Segundo Cross e Prussak (2002), essas relações são muitas vezes ignoradas pelos gestores e, quando percebidas, elas são consideradas como uma falha da organização, o que é entendido pelos autores como um erro.

Conde e Farias Filho (2016) consideram as relações informais as mais importantes, pois levam a melhorias dos processos e colaboram com os gestores no enfrentamento de impedimentos da estrutura formal e na disseminação do conhecimento na organização, evitando, por exemplo, o surgimento de lacunas de conhecimento (QIU; WANG; NIAN, 2014). Para os autores, essa informalidade decorre das relações sociais nas empresas, caracterizadas pela casualidade e espontaneidade, ao contrário das relações formais, caracterizadas por regras e procedimentos.

INTERAÇÕES, Campo Grande, MS, v. 22, n. 3, p. 941-957, jul./set. 2021. 
Garcia (2012) entende que, ao desconsiderarem-se as relações informais, a rede organizacional incorrerá na possibilidade de diminuição competitiva, pois importantes recursos intangíveis circulam nestas relações informais, especialmente o conhecimento.

Para que ocorra o alinhamento desejado entre as perspectivas formais e informais, é necessária uma integração para obter melhores resultados para a organização (CROSS; PRUSAK, 2002), uma vez que, para os gestores obterem o conhecimento desejado nas redes sociais, deve haver uma interação e distribuição social e funcional adequadas, as quais ajudem na superação de barreiras impostas pela estrutura hierárquica formal e funcional (CONDE; FARIAS-FILHO, 2016), que pode ser mais ou menos centralizada.

Existe um paradoxo a ser observado na estrutura de redes, pois, enquanto uma estrutura mais centralizada impõe maior controle e eficiência no uso de recursos (CUNLIFFE, 2008), estruturas mais descentralizadas contribuem para uma maior autonomia, o que leva a maior criatividade, inovação, flexibilidade e motivação (CONDE; FARIAS-FILHO, 2016).

Embora assuma-se a existência dessa ambiguidade, a essência de uma rede organizacional é a relação entre seus membros (BALESTRIN; VERSCHOORE; REYES JR., 2010), e os acordos relacionais existentes entre eles (CÂMARA et al., 2018; CAPALDO, 2007). Em um modelo cooperativo de gestão de rede, portanto, é necessário direcionar as ações para favorecerem o compartilhamento de conhecimento (TSAI, 2001), conduta que pode tornar a gestão mais eficiente.

Dhanaraj e Parkhe (2006) e Roth et al. (2012) entendem que as estratégias a serem adotadas devem considerar as características das redes e ser definidas coletivamente, buscando um consenso, ou podem conceder maior autonomia aos gestores da rede (ROTH et al., 2012).

A disseminação do conhecimento acontece em situações em que a empresa coopera com outras empresas (QIU; WANG; NIAN, 2014) por estruturação de relações de governança formal e informal (CÂMARA et al., 2018) e gestão focada em maximizar a transferência de informações e recursos intangíveis, como conhecimento técnico, aumento da competitividade e desempenho de membros da rede (GARCIA, 2012).

Essa desejada disseminação do conhecimento depende de diversos fatores, como o incentivo à equipe, que aumentará a sociabilidade; e uma coordenação eficiente das atividades, que reduzirá os limites da estrutura funcional, resultando na satisfação dos funcionários (CONDE; FARIAS-FILHO, 2016).

Portanto, de acordo com a forma como as redes estão estruturadas, naturalmente elas sofrerão variações em seus desempenhos enquanto redes, e as lacunas de conhecimento, a serem exploradas a seguir, poderão ser um problema a ser sanado.

\subsection{Rede organizacional e as lacunas de conhecimento}

Uma definição aceita de uma rede organizacional é aquela que afirma tratar-se de uma estrutura não linear, caracterizada pela descentralização, flexibilidade, dinamicidade, e que seja auto-organizável, estabelecendo-se principalmente por relações horizontais de colaboração (TOMAÉL; ALCARÁ; DI CHIARA, 2005).

Uma rede é um dos modelos organizacionais mais adequados para se obter conhecimento e transformá-lo em inovações, e o acúmulo deste conhecimento é o principal resultado da interação entre empresas em uma rede, devido à relação direta e positiva entre interações para trocar informações com outras (TOMAÉL; ALCARÁ; DI CHIARA, 2005). No entanto, muitas vezes, este processo revela a existência de lacunas no conhecimento gerado nas redes organizacionais.

INTERAÇÕES, Campo Grande, MS, v. 22, n. 3, p. 941-957, jul./set. 2021. 
Essas chamadas lacunas de conhecimento ocorrem quando o recurso de conhecimento intangível da rede não atende às necessidades específicas do ator (QIU; WANG; NIAN, 2014). Para os autores, estas lacunas surgem e se fortalecem a partir de uma distribuição desigual e dispersa do conhecimento gerado, possíveis de serem observadas nas situações em que empresas pertencentes a uma determinada rede não usufruírem do conhecimento gerado internamente, enquanto outras, pertencentes a esta mesma rede, fazerem uso total deste conhecimento.

Qiu, Wang e Nian (2014) desenvolveram um importante estudo para preencher lacunas de conhecimento usando o método tree-edit-distance. O algoritmo desenvolvido pelos autores, embora estivessem em uma rede intraorganizacional, é relevante para estudos em redes interorganizacionais. Para os autores, para cada tipo de lacuna de conhecimento, estratégias de preenchimento mais adequadas devem ser utilizadas.

Em situações em que as empresas de rede têm o conhecimento necessário, mas há lacunas de conhecimento na rede, Qiu, Wang e Nian (2014) enfatizam que o conhecimento pode ser mais bem distribuído por meio das seguintes etapas: i) mineração, ii) organização e conhecimento e iii) aprendizagem organizacional.

Em uma situação de distribuição desigual e dispersa do conhecimento organizacional, a gestão da rede deve criar o mapa do conhecimento e melhorar a capacidade de compartilhar e disseminar conhecimento (CONDE; FARIAS-FILHO, 2016; QIU; WANG; NIAN, 2014).

Em situações em que a rede tem parte do conhecimento necessário, mas não o suficiente para desenvolver produtos inovadores, Qiu, Wang e Nian (2014) sugerem a construção de alianças cooperativas. É comum em cooperações dentro de redes a existência de atividades de Pesquisa e Desenvolvimento (P\&D), aquisição e licenciamento de patentes conjuntas (CÂMARA et al., 2018), estratégias também destinadas à redução de riscos e custos.

Nos casos em que a empresa não possui o conhecimento necessário, como melhor maneira de preencher esse tipo de lacuna de conhecimento, Qiu, Wang e Nian (2014) propuseram a adoção e o desenvolvimento de capacidades absortivas, que envolvem processos de aquisição, seleção, transformação do conhecimento e externalização de produtos inovadores.

Além da consideração da necessidade de adaptação da estratégia à lacuna de conhecimento, Choo (2006) afirma que o conhecimento está relacionado à informação de forma cíclica, quando a necessidade de informação gera a busca de informações e, finalmente, o uso das informações e a fonte da criação de conhecimento de empresas individuais (Figura 1).

Figura 1 - Ciclo de informação e conhecimento

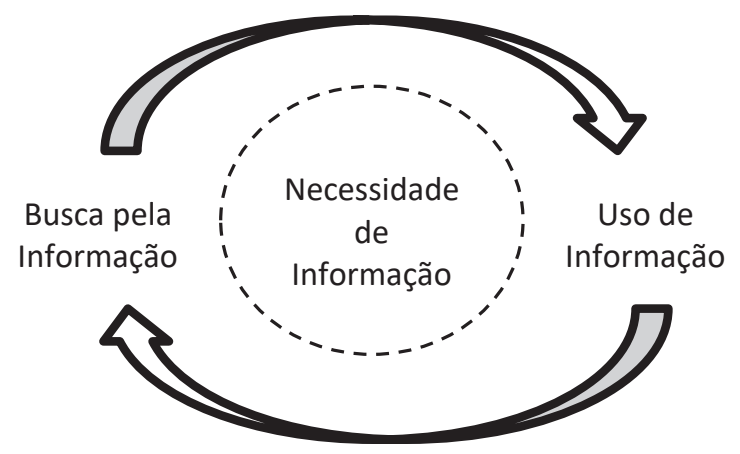

Fonte: Adaptado de Choo (1998). 
Para sugerir que a gestão considere o contexto do uso das informações, Choo (2006) entende a importância do cenário organizacional e dos componentes que formam o processo de comunicação intraorganizacional (VIEIRA, 2014). Em estudo baseado nas definições estabelecidas por Choo (2006), Vieira (2014) alerta para a relação cíclica e de dependência entre a necessidade e a utilização das informações.

Uma série de benefícios individuais pode ser obtida adotando o compartilhamento de informações em uma rede organizacional, dependendo da competitividade de cada ator, embora, para Qiu, Wang e Nian (2014), o principal ganho se deva ao desenvolvimento de novos produtos e ao preenchimento de lacunas de conhecimento.

Tomaél, Alcará e Di Chiara (2005) propõem que a interação é fundamental para a inovação, pois as empresas dificilmente inovam sozinhas. Para os autores, as empresas precisam das fontes de conhecimento que ocorrem por meio da interação de múltiplos agentes (dentro ou fora da organização), desde que a empresa que recebe conhecimento seja capaz de obtê-lo modificado ou ampliá-lo.

Nesse sentido, Tomaél, Alcará e Di Chiara (2005) destacam que, quando se flexibilizam as relações entre pessoas, redes, em seus diferentes níveis e aplicações, aumenta-se o compartilhamento de informações entre organizações e indivíduos e, consequentemente, geram-se conhecimento e inovação tecnológica, apresentando-se, portanto, como mecanismos indispensáveis para a melhoria dos recursos organizacionais e o incremento da capacidade tecnológica na geração de inovações.

Para que se otimize a utilização dos recursos e da capacidade tecnológica em um ambiente de rede, Pelz, De Jesus e Raabe (2015) apontam o método tree-edit-distance. O referido método é utilizado em muitas pesquisas em ciência da computação, para determinar, por exemplo, quantos passos são necessários para transformar uma árvore em outra, aplicando um conjunto de operações, como inserir, substituir e remover nós (PELZ; DE JESUS; RAABE, 2015).

Em seu estudo, Qiu, Wang e Nian (2014) apresentam três contribuições relevantes para o estudo da inovação organizacional: a construção de um mapa do conhecimento, uma busca por parceiros mais identificáveis e uma identificação de lacunas de conhecimento. Essas contribuições são fundamentais para a adoção de estratégias colaborativas e para o desenvolvimento de produtos inovadores.

\section{METODOLOGIA}

O estudo é classificado como descritivo, pois descreve como as relações ocorrem entre as medidas de centralidade das empresas e as estratégias cooperativas dos gestores (GIL, 2006) pertencentes a um instituto de tecnologia (IT) na região do $A B C$, região metropolitana de São Paulo, o qual teve sua identificação omitida e substituída pelo nome fictício EBT.

A EBT foi criada em 2007 por empresários da região do ABC Paulista em setores como automação, gestão de TI e Telecom. O objetivo da EBT visa aumentar os negócios para empresas, receita para o município e emprego para os cidadãos.

A EBT tem valores, associativismo, cooperação, empreendedorismo, inovação e ética. Contava, no período da coleta de dados, com 48 associados e 19 parceiros, que incluem universidades, associações e um grupo de anjos adotivos.

Em relação ao delineamento da pesquisa, ela classifica-se como uma survey, pois questiona diretamente as empresas do instituto (GIL, 2006). O questionário foi disponibilizado por meio do 
Google Forms para 40 gestores de empresas, entre 15 de maio e 9 de julho de 2018, recebendo um retorno de 33 entrevistados (78,57\%).

O questionário foi estruturado em três partes. A primeira parte consistiu no consentimento do entrevistado para participar da pesquisa, ou seja, o aceite no Termo de Consentimento Livre e Esclarecido (TCLE). A segunda parte capturou as identificações do entrevistado (nome; sexo; Registro Geral [RG]); data e local de nascimento; telefone; endereço; e e-mail) e da empresa (razão social; cargo que ocupa na empresa; tempo de associação da empresa ao EBT; e ramo de atuação). A partir das respostas dos dados da empresa, foi possível analisar os atributos associados (se ocupam cargo na direção do EBT e qual cargo). Na terceira parte, duas perguntas foram feitas, sendo uma fechada e outra aberta.

A pergunta fechada foi para verificar com qual(is) das demais empresas aquela obtinha conhecimento ou complementaridade por meio da interação. Junto à pergunta, foi apresentada uma lista com todas as empresas associadas ao EBT. A pergunta aberta foi uma consulta de qual sugestão o entrevistado tinha para ser implementada na EBT, para que se consiga interagir e inovar. Com estas respostas, seria possível identificar relações cooperativas entre empresas e as fontes de conhecimento ou complementaridade por meio da interação entre a empresa e as demais. Os dados foram analisados com o uso do software IBM SPSS Statistics 23, UCINET 6 e Netdraw.

Para alcançar o objetivo, utilizou-se uma análise multivariada, sendo aplicada ARS, para se compreender como ocorre a relação entre os atores. A técnica estatística Análise Univariada da Variância (ANOVA) foi usada para testar se a média da inovação dos gestores das empresas que têm algum cargo na direção do instituto é diferente da média dos gestores que não têm nenhum cargo de direção.

Outra medida verificada foi a núcleo-periferia (Core/Periphery), que consiste em duas classes de nós, em que, em uma parte os nós estão altamente interconectados, formando um bloco coeso e uma classe de atores que estão mais fracamente conectados ao bloco coeso (EVERETT; BORGATTI, 1999). Os autores complementam que, na teoria núcleo-periferia, existe uma matriz núcleo-periferia ideal, que ocorre quando os nós centrais são adjacentes a outros nós centrais e a alguns nós periféricos, enquanto os nós periféricos não estão conectados a outros nós periféricos.

\subsection{Análise dos dados}

A análise dos dados foi desenvolvida segundo as propostas de Conde e Farias Filho (2016), que sugerem a ARS para estudos desta natureza, e Bacharach (1989), que entende a necessidade de essas pesquisas também abordarem testes estatísticos.

Após a apresentação de lista com as empresas participantes do EBT, a rede foi construída com base na seguinte pergunta: com quais empresas a seguir a sua empresa obteve conhecimento e o utilizou para fins comerciais?

Com base na pergunta feita, cada entrevistado atribuiu notas às empresas com as quais atuou cooperativamente e obteve conhecimento, que efetivamente criou um produto inserido no mercado. Esta questão sobre a obtenção de conhecimento e seu uso para fins comerciais buscou identificar a interação entre as empresas para desenvolver a implementação da cooperação. Outra pergunta realizada na survey foi uma questão aberta em que os gestores poderiam expor suas ideias de melhoria para o EBT. A Tabela 1 mostra as empresas e os atributos: tempo associado em anos, a principal atividade realizada pela empresa e a função do gestor no EBT. 
Tabela 1 - Perfil das empresas

\begin{tabular}{|c|c|c|c|}
\hline Empresa & Tempo & Atividade & Função \\
\hline E1 & 2 & Sinalização horizontal e vertical & Vice-Presidente \\
\hline E2 & 4 & Consultoria em Gestão e TIC & Diretoria de $\mathrm{RH}$ \\
\hline E3 & 1 & Consultoria em Gestão e TIC & Nenhum \\
\hline E4 & 1 & Desenvolvimento de Software para Gestão e TIC & Diretoria de BPO \\
\hline E5 & 1 & Projetos de arquitetura/construção civil & Nenhum \\
\hline E6 & 5 & Segurança de internet/rede & Diretoria de Empreendedorismo \\
\hline E7 & 2 & Segurança de patrimônio & Presidente \\
\hline E8 & 0 & Educação & Diretoria da Mulher \\
\hline E9 & 1 & Desenvolvimento de software & Diretoria de SmartBuild \\
\hline E10 & 1 & Educação & Diretoria de EdTech \\
\hline E11 & 2 & Soluções para cidades inteligentes & Diretoria de SmartCity \\
\hline $\mathrm{E} 12$ & 2 & Consultoria em Gestão e TIC & Diretoria de Inovação \\
\hline E13 & 1 & Coaching & Diretoria de Estratégia Financeira \\
\hline E14 & 2 & Educação & Diretoria Jovem \\
\hline E15 & 2 & Marketing digital & Assessoria de Imprensa \\
\hline E16 & 1 & Coworking & Nenhum \\
\hline E17 & 1 & Desenvolvimento de soluções customizadas & Nenhum \\
\hline E18 & 2 & $\begin{array}{c}\text { Solução para arquitetos, engenheiros e } \\
\text { decoradores }\end{array}$ & Nenhum \\
\hline E19 & 2 & Coworking & Diretoria de Networking \\
\hline E20 & 1 & Consultoria em Gestão e TIC & Tesouraria \\
\hline E21 & 2 & Consultoria em Gestão e TIC & Diretoria de Educação \\
\hline E22 & 10 & $\begin{array}{c}\text { Ferramentas para desenvolvimento } \\
\text { eletroeletrônico }\end{array}$ & Nenhum \\
\hline E23 & 2 & Desenvolvimento de soluções customizadas & Nenhum \\
\hline E24 & 3 & Recuperação de crédito & Nenhum \\
\hline E25 & 3 & Contabilidade & Diretoria Contábil \\
\hline E26 & 2 & Desenvolvimento de software & Diretoria de Técnologia \\
\hline E27 & 2 & Marketing digital & Gerência Executiva \\
\hline E28 & 1 & Projetos de arquitetura/Construção ivil & Nenhum \\
\hline E29 & 10 & Desenvolvimento de Software para Gestão e TIC & Sócio Fundador \\
\hline $\mathrm{E} 30$ & 2 & Fabricação de ferramentas & Diretoria de Ind. 4.0 \\
\hline E31 & 2 & Desenvolvimento de soluções customizadas & Nenhum \\
\hline E32 & 4 & $\begin{array}{c}\text { Segurança de internet/rede } \\
\end{array}$ & Nenhum \\
\hline E33 & 10 & Desenvolvimento de Software para Gestão e TIC & Sócio Fundador \\
\hline
\end{tabular}

Fonte: Elaboração dos autores.

A rede de obtenção de conhecimentos apresentada na Figura 2, por meio da Análise de Redes Sociais, proporciona uma análise visual da cooperação entre os gestores da rede. Verificase que a empresa E6 coopera com 22 empresas e caracteriza-se não só pelo elevado número de grau de centralidade, como também por interagir com cinco empresas que só cooperam com ela (E11, E21, E23, E24 e E33). A empresa E6 é associada ao EBT há 5 (cinco) anos, atua no ramo de segurança de internet e seu membro respondente da pesquisa faz parte da Diretoria de Empreendedorismo do EBT.

A Figura 2 é dividida em quadrados e círculos que são os atributos dos associados. Foram utilizados os atributos tempo de vinculação ao instituto, atividade principal e cargo de direção 
(presidência, vice-presidência, diretorias administrativa ou técnica) que exerce no EBT. Enquanto os quadrados apresentam as empresas que têm algum cargo executivo ou técnico no EBT, o círculo mostra as empresas que, embora sejam associadas, não participam na gestão da rede.

A supracitada figura revela ainda que as empresas que detêm cargos de diretoria no EBT são em maior número e detêm uma centralidade maior na rede para obter conhecimentos, enquanto as empresas que são somente associadas estão localizadas na periferia, sem nenhum vínculo ou com poucas vinculações que envolvam transferência de conhecimento.

Faz-se relevante mencionar que a diferença na centralidade pode ser consequência dos seus atributos, como a questão do tempo de vinculação ao EBT. Por exemplo, na empresa E6, o gestor ocupa cargo de direção, tem cinco anos de vinculação ao EBT e apresenta um alto grau de centralidade. As empresas E11 e E21 também possuem gestores ocupando cargos na direção do EBT, mas com pouco tempo como associados (apenas 2 anos) e apresentando um baixo grau de centralidade.

Uma contraposição foi percebida na E33, uma vez que ela não tem nenhum executivo ocupando cargo no EBT, mas é um dos associados mais antigos, sendo, inclusive, um fundador do EBT, com mais de 10 anos de vinculação, e apresenta um baixo grau de centralidade.

As empresas E23 e E24 também possuem baixo grau de centralidade, não têm gestores ocupando cargos no EBT, além do baixo tempo de vinculação, 2 e 3 anos, respectivamente, como associados. Baixo grau significa poucos contatos para obter conhecimento de outras empresas com fins comerciais.

Figura 2 - Transferência de conhecimento na rede

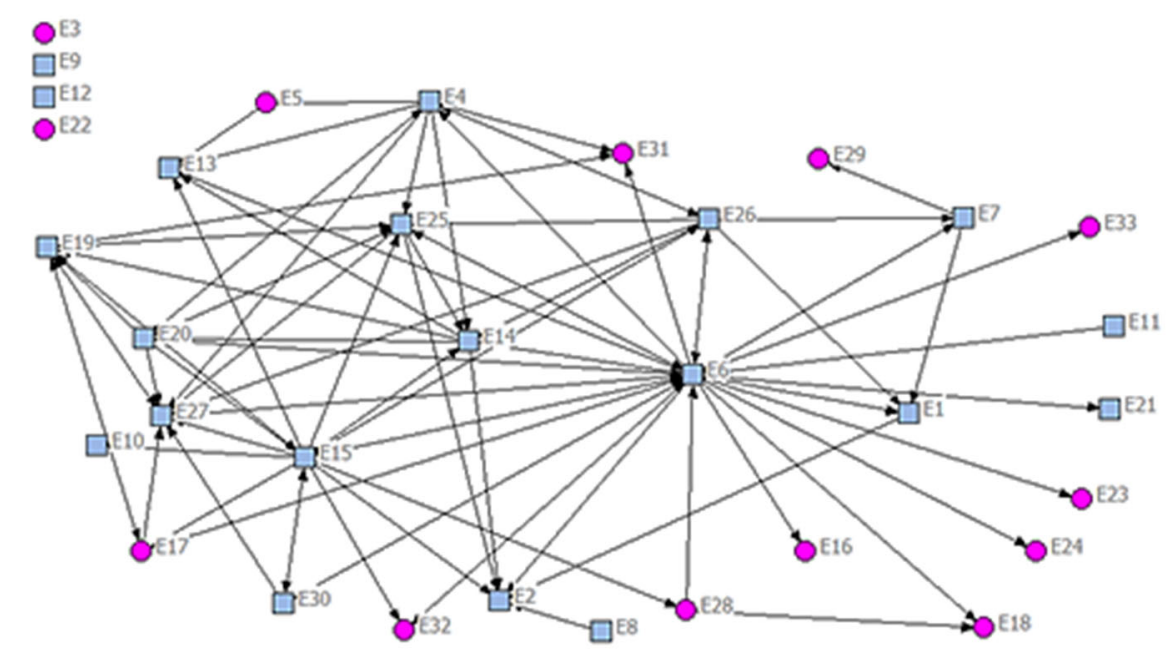

Fonte: Elaboração dos autores.

Alguns aspectos devem ser considerados, a partir desta análise. Inicialmente, não se pode confirmar uma relação entre o tempo de associação das empresas no EBT e o grau de centralidade, apenas entre in-degree e out-degree.

A Figura 3 apresenta a análise da centralidade, em que se entende que a empresa E6 não só transmite (out-degree) conhecimento para 20 empresas associadas como também é beneficiada (in-degree) por conhecimento recebido (10 empresas), tornando-se uma empresa atuante e de grande importância para a disseminação do conhecimento e geração de inovação na rede do 
instituto. Hanneman e Mark (2005) apresentam um ponto de vista sui generis, ao sugerirem que, enquanto o out-degree pode indicar influência sobre os demais atores, o in-degree parece demonstrar um desejo dos outros integrantes da rede de influenciar, embora esta visão peculiar seja contrariada por outros autores.

Esta visão contrária pode ser verificada na opinião de Tortoriello, Reagans e McEvely (2012), já que eles consideram que a alta centralidade de informações de um indivíduo em uma rede significa maior propensão de eles receberem informações de menor valor, pois a condição de centralidade o expõe tanto para informações valorosas como também para informações sem relevância.

Em relação às atividades, embora exista uma grande diversidade, não se pode afirmar que exista um conhecimento complementar entre estas empresas, o que poderia facilitar a transferência de conhecimento na rede. Nem mesmo o fato de ocupar ou não um cargo de direção no instituto parece relacionar-se com a posição da empresa na rede, sendo necessária a utilização das medidas adicionais, Tabela 2: Matriz de densidade Core/Periphery e ANOVA.

Na análise de Hanneman e Mark (2005), empresas em posição de out-degree se encontram favorecidas, na medida em que podem extrair melhores barganhas nas trocas e ter maior influência, enquanto nas empresas na posição in-degree, as demais empresas, em um ato de deferência ou reconhecimento, entendem valer a pena a tentativa de influenciá-las.

Figura 3 - Gráfico de barras de in-degree e out-degree

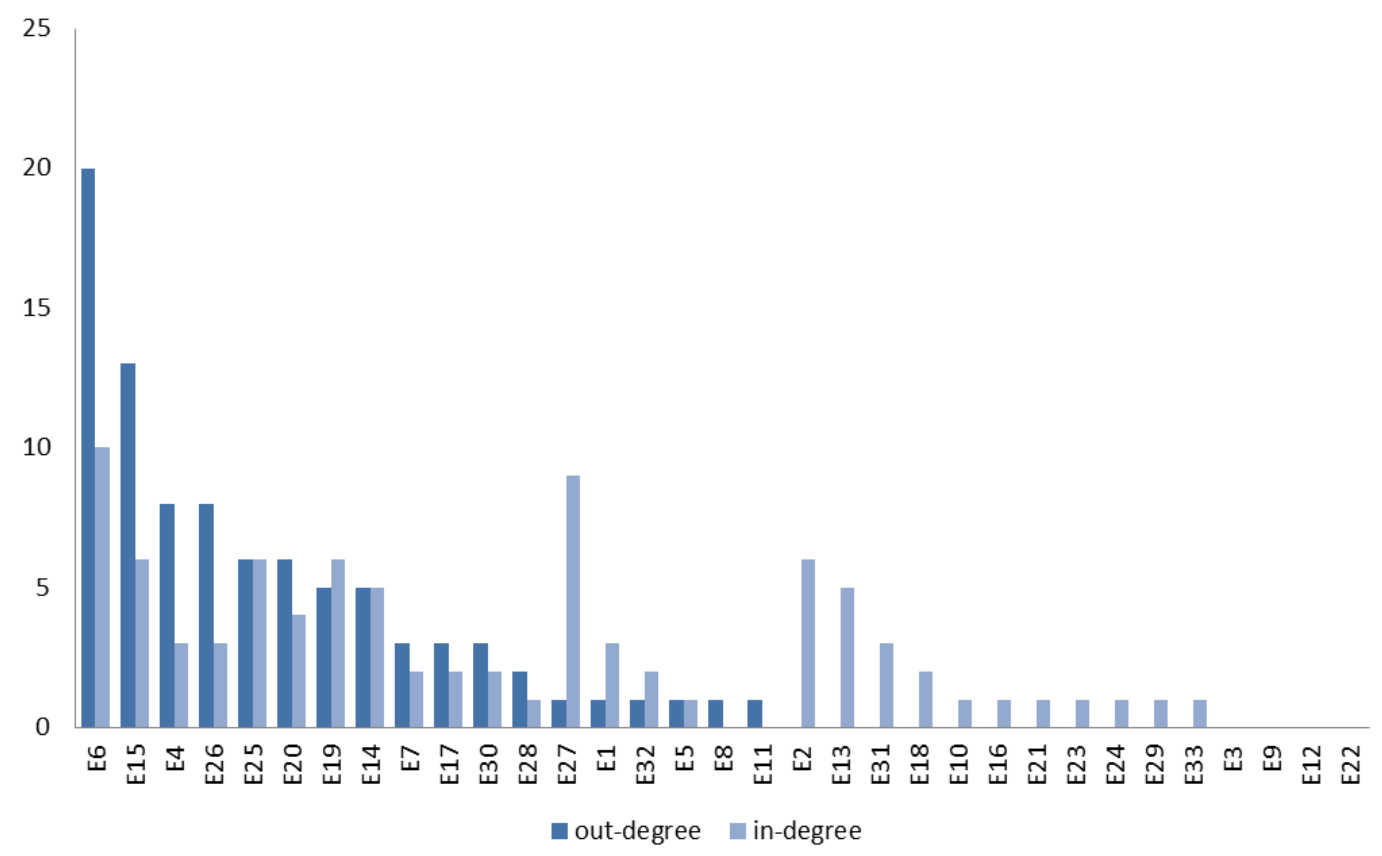

Fonte: Elaboração dos autores.

Além da empresa E6, as empresas E2 e E14 se mostraram também altamente relacionadas com os demais integrantes do EBT. E2 é uma empresa de consultoria em recursos humanos, já faz parte do instituto há 4 anos e relaciona-se com as seguintes empresas: E29, E19, E9, E12, E6, E7, E26, E27, E25, E15, E13 e E8; e seu gestor ocupa cargo de direção no instituto.

E14 está associada há 2 anos, trabalha com treinamentos, relaciona-se com as seguintes empresas: E33, E6, E2, E7, E26, E14, E27, E25, E15, E4, E13, E20, E10 e E16. Da mesma forma que a empresa anterior, seu gestor ocupa cargo de direção no instituto. 
A densidade da rede é obtida pelo quociente das relações que efetivamente se consolidaram e da quantidade de relações possíveis entre os atores, ou seja, de 100\% dás relações que foram efetivadas. Ao considerarmos as 33 empresas que responderam ao questionário, obtêm-se 1.056 possíveis relações, mas, uma vez que só foram realizadas 88 interações, encontra-se uma densidade de $8,33 \%$; portanto, muito baixa, quando se considera a totalidade de possíveis relações. Isto demonstra que a rede é carente de atores que buscam conhecimento com outras empresas do EBT com fins comerciais.

Na Tabela 2, elabora-se a matriz Core/Periphery, que apresentou um ajuste de correlação (fit correlation $)=0,6864$. No quadrante superior esquerdo, encontram-se empresas centrais que obtêm conhecimentos de empresas igualmente centrais, representando laços para a obtenção de conhecimentos entre as empresas centrais com elas mesmas.

No quadrante inferior direito, encontram-se as empresas periféricas que obtêm conhecimentos das demais empresas periféricas, representando laços para a obtenção de conhecimentos entre as empresas periféricas com elas mesmas.

No quadrante superior direito, estão as empresas centrais, as quais obtêm conhecimentos provenientes das empresas periféricas, o que representa a transferência de conhecimento das empresas periféricas para as empresas centrais.

No quadrante inferior esquerdo, estão as empresas periféricas, as quais obtêm conhecimentos das empresas centrais, o que representa a transferência de conhecimento das empresas centrais para as empresas periféricas.

Por meio da matriz Core/Periphery, identificaram-se como empresas centrais: E4, E6, E14, E15, E19, E20, E25, E26, E27, enquanto as periféricas foram: E1, E2, E3, E5, E7, E8, E9, E10, E11, E12, E13, E16, E17, E18, E21, E22, E23, E24, E28, E29, E30, E31, E32 e E33, confirmando os achados da Tabela 2, que apresenta a rede social de transferência de conhecimento.

Ao trazer à tona o questionamento que gerou a matriz - com quais empresas a seguir a sua empresa obteve conhecimento e o utilizou para fins comerciais? -, percebe-se que as empresas centrais (E4, E6, E14, E15, E19, E20, E25, E26, E27), na sua totalidade formada por diretores do EBT, relacionam-se fortemente entre elas. Pôde-se verificar que empresas centrais formadas tanto por aquelas que possuem cargos como aquelas que não possuem função na direção no instituto transmitem conhecimento para empresas periféricas, mas em um volume bem menor (Tabela 2). Finalmente, os quadrantes 3 e 4, formados por empresas periféricas (Tabela 2).

Este padrão de distribuição de conhecimento não é o que mais agrega valor à rede, uma vez que esta estrutura Core/Periphery apresenta elevada assimetria de conhecimento pela grande diferença de médias entre blocos (EVERETT; BORGATTI, 1999).

Em seguida, estimou-se a densidade de cada quadrante, para poder fazer inferências sobre a relação entre as empresas centrais, que têm como atributo relevante o cargo que seus representantes ocupam no EBT, e as periféricas, formadas predominantemente por empresas que são apenas associados (Tabela 2).

Tabela 2 - Matriz de densidade Core/Periphery

\begin{tabular}{|c|c|c|}
\hline & Core & Periphery \\
\hline Core & 0.583 & 0.139 \\
\hline Periphery & 0.046 & 0.011 \\
\hline
\end{tabular}

Fonte: Elaboração dos autores. 
Como esperado, a maior densidade está no centro da rede, que é de 0,583\%, o que indica que, com base em todas as relações possíveis, 58,3\% são mantidas entre as empresas centrais com elas mesmas. Das empresas do centro, 58,3\% obtiveram conhecimento das outras do centro, o qual foi utilizado para fins comerciais.

A interface núcleo/periferia mostra uma medida de densidade de 0,139. Essa densidade $(13,9 \%)$ confirma que as empresas centrais obtêm também informações de empresas periféricas, embora em quantidades menores.

Considerando a predominância de empresas que possuem gestores atuando como diretores do EBT, sugere-se que esse atributo explica, em grande parte, a concentração de conhecimento entre diretores e a direção que o conhecimento segue dentro do EBT.

As relações periférico-centro e periférico-periférico apresentam uma densidade quase inexistente $(0,046$ e 0,011 respectivamente), confirmando que as empresas que são apenas associadas influenciam muito pouco as empresas que têm gestores com cargo no instituto $(4,6 \%)$. E, de maneira ainda mais clara, verifica-se a pouca concentração de conhecimento entre as empresas periféricas (1,1\%). Essas densidades significam, também, que estas empresas praticamente não obtêm conhecimento entre elas para fins comerciais.

Por meio da ANOVA, testou-se a diferença média na interação, visando à inovação entre empresas que têm executivos ocupando cargo de diretor no EBT, em que foi atribuído a ele o label 1; para o presidente e vice-presidente do EBT, foi atribuído o label 2; e, para os que não ocupam nenhum cargo no instituto, ou seja, atuam apenas como associados, foi atribuído label 0.

Os cargos de direção e, principalmente, presidente, podem levar a uma centralidade do ator pela participação mais efetiva no EBT ou por algum dos atores ser diretor de uma área que relaciona um grande número de atores. Por exemplo, espera-se que a E19, que ocupa o cargo de diretora de Networking - ou seja, tem a função de conectar os atores da rede -, detenha um grande volume de interações com os demais associados, função que poderia facilmente convergir para um maior volume de troca de conhecimentos.

Enquanto a variável independente (o fato de ele pertencer ou não à gestão do EBT) foi obtida a partir da identificação da participação dos atores na gestão do EBT, a variável dependente (interação por complementaridade para inovação) foi obtida pela quantidade de interações que cada empresa tem na rede.

Ao realizar-se a ANOVA, encontrou-se uma significância global de 0,012. Optou-se pela rejeição da hipótese de igualdade das variâncias e concluiu-se que existe diferença entre ser da diretoria, da presidência e apenas associado com relação à variável inovação (Tabela 4), obtida por meio da nota atribuída.

Tabela 3 - Resultados da ANOVA

\begin{tabular}{|c|c|c|c|c|c|}
\hline & Soma de quadrados & Grau de Liberdade (GL) & Mean Square & F & Sig. \\
\hline Entre grupos & 68.721 & 2 & 34.361 & 5.115 & 0.012 \\
\hline Intra grupos & 201.521 & 30 & 6.717 & & \\
\hline Total & 270.242 & 32 & & & \\
\hline
\end{tabular}

Fonte: Elaboração dos autores.

O teste de Bonferroni concluiu que ser presidente no EBT não mostrou importância. Os resultados sugerem que, relacionado à inovação, é indiferente se o gerente da empresa ocupa o 
cargo de diretor, presidente, vice-presidente ou ex-presidente; ou não tem um cargo ou função em EBT e é presidente, vice-presidente ou ex-presidente. Contudo eles mostram significado entre ter uma posição na direção ou ser apenas associado.

\section{CONSIDERAÇÕES FINAIS}

Por meio da análise da densidade da rede, verificou-se sua baixa densidade, bem como as poucas interações centradas no ator, especialmente entre aqueles que ocupam um cargo de diretor no EBT. O cargo ocupado pelo diretor do EBT, portanto, é o de intermediação, pois a posição que o ator ocupa detém o poder de conectar-se a outros atores, tornando-se a terceira parte nas relações entre os atores (CONDE; FARIAS-FILHO, 2016). Essa conexão de outros diretores com os gestores de outras empresas pode disseminar ou restringir o conhecimento na rede, utilizar a circulação desses recursos para melhor identificar oportunidades e melhores recursos disponíveis diretos (CONDE; FARIAS-FILHO, 2016; HANNEMAN; MARK, 2005; TSAI, 2001).

Ao considerar o trabalho de Dhanaraj e Parkhe (2006), que dá às empresas o papel central da liderança e o poder de associar recursos e liderar a rede, essas empresas parecem ter uma postura cooperativa, embora incipiente, na promoção do conhecimento, condição necessária para o desenvolvimento e a capacidade inovadora de outras empresas de rede. Essa falta de troca de conhecimento ocorre devido a um contexto interno que não permite saber quais recursos estão disponíveis e onde estão sendo armazenados (CONDE; FARIAS-FILHO, 2016). Portanto é necessário reduzir a centralidade para proporcionar maior colaboração entre os participantes e o consequente aumento da capacidade inovadora das empresas.

Ao considerar a questão aberta da pesquisa, em que os gestores poderiam expor suas ideias de melhoria para o instituto, os atores sugeriram a adoção de mecanismos de gestão para o aprimoramento da rede do EBT. Concluiu-se a necessidade de se adotarem estratégias orientadas ao conhecimento, a fim de se evitar lacunas (QIU; WANG; NIAN, 2014).

Ainda por meio das respostas à questão aberta, foi revelado que há lacunas no conhecimento, portanto é necessário adotar a estratégia mais adequada para a rede. Ao perceber que a rede tem o conhecimento técnico necessário, mas as lacunas de conhecimento persistem, entende-se a necessidade de construir uma rede organizacional focada na relação entre seus membros (BALESTRIN; VERSCHOORE; REYES JR., 2010), fortalecendo acordos relacionais entre os membros (CÂMARA et al., 2018; CAPALDO, 2007) e um modelo de gestão de aprendizagem organizacional.

Uma sugestão para futuras pesquisas seria realizar um estudo no Brasil com base em Tsai (2001), com o objetivo de verificar a relação entre desempenho e inovação em dois institutos de pesquisa, com alta e baixa capacidade absortiva, por meio de uma ARS.

Uma limitação do estudo é que apenas foi identificada a existência de lacunas de conhecimento e o desenvolvimento de estratégias, mas não foi usado o algoritmo proposto pelos Qiu, Wang e Nian (2014) para identificar as empresas mais adequadas para se compartilhar conhecimento.

\section{REFERÊNCIAS}

AHUJA, G. Collaboration networks, structural holes and innovation: a longitudinal study. Administrative Science Quarterly, New York, v. 45, n. 3, p. 425-55, 2000. 
BACHARACH, S. B. Organizational theories: some criteria for evaluation. Academy of Management Review, New York, v. 14, n. 4, p. 496-515, 1989.

BALESTRIN, A.; VERSCHOORE, J. R.; REYES JR., E. O campo de estudo sobre redes de cooperação interorganizacional no Brasil. RAC, Curitiba, v. 14, n. 3, p. 458-77, 2010.

BRASS, D. J.; BURKHARDT, M. E. Potential power and power use: an investigation of structure and behavior. Academy of Management Journal, New York, v. 36, n. 3, p. 441-70, 1993.

BRESCHI, S.; MALERBA, F. Clusters, networks and innovation. Oxford: Oxford University Press, 2005.

CÂMARA, S. F.; DE LIMA, B. B.; MOTA, T. L. N. G.; SILVA, A. L. The management of innovation networks: possibilities of collaboration in light of game theory. Business and Management Studies, Beaverton, v. 4, n. 2, p. 24-34, 2018.

CAPALDO, A. Network structure and innovation: the leveraging of a dual network as a distinctive relational capability. Strategic Management Journal, London, v. 28, n. 6, p. 585-608, 2007.

CHOO, C. W. A organização do conhecimento: como as organizações usam a informação para criar significado, construir conhecimento e tomar decisões. 2. ed. Tradução de Eliana Rocha. São Paulo: Editora Senac, 2006.

$\mathrm{CHOO}, \mathrm{C}$. W. The knowing organization: how organizations use information for construct meaning, create knowledge and make decisions. Nova York: Oxford Press, 1998.

CONDE, R. N. C.; FARIAS-FILHO, M. C. Relações informais influenciadas pela estrutura formal: uma análise de redes sociais de gestores. RCA, Florianópolis, v. 1, n. 3, p. 68-80, 2016.

COSTA, E. S.; BITANTE, A. P.; BRITTO, L. C.; PINHEIRO, L. R. D.; FARINA, M. C. Análise das relações e ações conjuntas entre as empresas do APL têxtil da região metropolitana de São Paulo: contribuições para o seu crescimento. Interações, Campo Grande, v. 19, n. 2, p. 401-15, 2018.

CROSS, R.; PRUSAK, L. The people who make organizations go-or stop. Harvard Business Review, Cambridge, v. 80, n. 6, p. 104-12, 2002.

CUNLIFFE, A. L. Organization theory. London: Sage, 2008.

DHANARAJ, C.; PARKHE, A. Orchestrating innovation networks. Academy of Management Review, New York, v. 31, n. 3, p. 659-69, 2006.

DYER, J. H.; NOBEOKA, K. Creating and managing a high-performance knowledge-sharing network: the toyota case. Strategic Management Journal, London, v. 21, n. 3, p. 345-67, 2000. Special issue: strategic networks

EVERETT, M. G.; BORGATTI, S. P. The centrality of groups and classes. The Journal of Mathematical Sociology, London, v. 23, n. 3, p. 181-201, 1999.

GARCIA, I. Uma visão antropológica das redes sociais. Harvard Business Review Brasil, [s.I.], n. 2, 12 jan. 2012.

GIL, A. C. Métodos e técnicas de pesquisa social. 6. ed. São Paulo: Atlas, 2006.

GRANDORI, A.; SODA, G. Inter-firm networks: antecedents, mechanisms and forms. Organization Studies, London, v. 16, n. 2, p. 183-214, 1995. 
GUEDES, H. P.; ZIVIANI, F.; PAIVA, R. V. C. P.; FERREIRA, M. A. T.; HERZOG, M. M. Mensuração da capacidade absortiva: um estudo nas empresas brasileiras fabricantes de coletores solares. Gestão \& Produção, São Carlos, v. 24, n. 1, p. 50-63, 2017.

HANNEMAN, R. A.; MARK, R. Introduction to social network methods. Riverside, CA: University of California, 2005. Disponível em: http://faculty.ucr.edu/ hanneman/. Acesso em: 23 jul. 2019.

HITT, M. A.; IRELAND, R. D.; HOSKISSON, R. E. Administração estratégica: competitividade e globalização. Tradução de All Tasks. 2. ed. São Paulo: Cengage Learning, 2011.

HOPPE, B.; REINELT, C. Social network analysis and the evaluation of leadership networks. Leadership Quarterly, Amsterdam, v. 21, n. 4, p. 600-19, 2010.

LEONARDO, S. B.; FARINA, M. C.; ANDREOLI, T. P.; LIMA, A. P. M. B. Relacionamentos interpessoais formal e informal: interação das redes no ambiente acadêmico. RAC, Curitiba, v. 23, n. 3, p. 395-415, 2019.

NOHRIA, N.; ECCLES, R. G. Networks and organizations: structure, form and action. Boston: Harvard Business School Press, 1992.

NOOTEBOOM, B.; GILSING V. A. Density and strength of ties in innovation networks: a competence and governance view. Tilburg: Econpapaers, 2005.

PELZ, F. D.; DE JESUS, E. A.; RAABE, A. L. A. Um mecanismo para correção automática de exercícios práticos de programação introdutória. In: SIMPÓSIO BRASILEIRO DE INFORMÁTICA NA EDUCAÇÃO [SBIE], 23., 2630 out. 2015, Maceió. Anais [...]. Maceió: SBIE, 2015.

POWELL, W. W.; KOPUT, K. W.; SMITH-DOERR, L. Interorganizational collaboration and the locus of innovation: networks of learning in biotechnology. Administrative Science Quarterly, London, v. 41, n. 1, p. 116-45, 1996.

QIU, J.; WANG, Z.; NIAN, C. An approach to filling firms' knowledge gaps based on organisational knowledge structure. Journal of Knowledge Management, London, v. 18, n. 1, p. 1-18, 2014.

ROTH, A. L.; WEGNER, D.; ANTUNES JÚNIOR, J. A.; PÁDUA, A. D. Diferenças e inter-relações dos conceitos de governança e gestão de redes horizontais de empresas: contribuições para o campo de estudos. Revista Administrativa, São Paulo, v. 47, n. 1, p. 112-23, 2012.

TOMAÉL, M. I.; ALCARÁ, A. R.; DI CHIARA, I. G. Das redes sociais à inovação. Ciência da Informação, Brasília, v. 34, n. 2, p. 93-104, 2005.

TORTORIELLO, M.; REAGANS, R.; MCEVILY, B. Bridging the knowledge gap: the influence of strong ties, network cohesion, and network range on the transfer of knowledge between organizational units. Organization Science, Catonsville, v. 23, n. 4, p. 1024-39, 2012.

TSAI, W. Knowledge transfer in intraorganizational networks: effects of network position and absorptive capacity on business unit innovation and performance. Academy of Management Journal, New York, v. 44, n. 5, p. 996-1004, 2001.

VIEIRA, E. A percepção da informação e da sua relevância no cenário institucional: sob a perspectiva de gestores e líderes. Cadernos EBAPE.BR, Rio de Janeiro, v. 12, n. especial, p. 534-52, 2014.

WASSERMAN, S.; GALASKIEWICZ, J. Advances in social network analysis. London: Sage, 1994. 
ZANCAN, C.; DOS SANTOS, P. C. F.; CAMPOS, V. O. As contribuições teóricas da análise de redes sociais (ARS) aos estudos organizacionais. Revista Alcance, Vale do Itajaí, v. 19, n. 1, p. 62-82, 2012.

\section{Sobre os autores:}

Milton Carlos Farina: Doutor em Administração pela Universidade de São Paulo (USP). Mestre em Administração de Empresas pela Fundação Getulio Vargas (FGV). Graduado em Matemática pela USP. Professor do programa de pós-graduação em Administração na Universidade Municipal de São Caetano do Sul (USCS) e dos cursos de graduação da mesma Instituição. E-mail: milton.farina@online.uscs.edu.br, Orcid: http://orcid.org/0000-0003-0551-8282

Rodrigo Santos de Melo: Doutor em Administração pela Universidade Municipal de São Caetano do Sul (USCS). Mestre em Administração pela Universidade Estadual do Ceará (UECE). Especialista em Administração Financeira pela UECE; e em Gestão Avançada pela Universidade de Coimbra (UC). Graduado em Administração pela Universidade de Fortaleza (UNIFOR). Professor do curso de Administração na Universidade Federal do Piauí (UFPI). E-mail: rodrigosantos@ufpi.edu.br, Orcid: https://orcid.org/0000-0003-3348-5326

Flávio Gálio Araújo Dutra: Doutorando em Administração na Universidade Municipal de São Caetano do Sul (USCS). Mestre em Educação, Administração e Comunicação pela Universidade São Marcos (USM). Pós-graduado em Gerência em Recursos Humanos pela Universidade Estadual do Ceará (UECE). Graduado em Administração de Empresas pela Universidade Federal do Piauí (UFPI). Atualmente, é professor na UFPI, lotado no Campus Amilcar Ferreira Sobral (CAFS), na cidade de Floriano. E-mail: flaviogalio@ufpi.edu.br, Orcid: http://orcid.org/0000-0003-0244-9269 
